\title{
Staging Airliner Service
}

\author{
Andrew S. Hahn ${ }^{*}$ \\ NASA Langley Research Center, Hampton, VA, 23681
}

There is a general consensus building that historically high fuel prices and greater public awareness of the emissions that result from burning fuel are going to be long-term concerns for those who design, build, and operate airliners. The possibility of saving both fuel and reducing emissions has rekindled interest in breaking very long-range airline flights into multiple stages or even adopting in-flight refueling. It is likely that staging will result in lower fuel burn, and recent published reports have suggested that the savings are substantial, particularly if the airliner is designed from the outset for this kind of operation. Given that staging runs against the design and operation historical trend, this result begs for further attention. This paper will examine the staging question, examining both analytic and numeric performance estimation methodologies to quantify the likely amount of fuel savings that can be expected and the resulting design impacts on the airliner.

\section{Nomenclature}

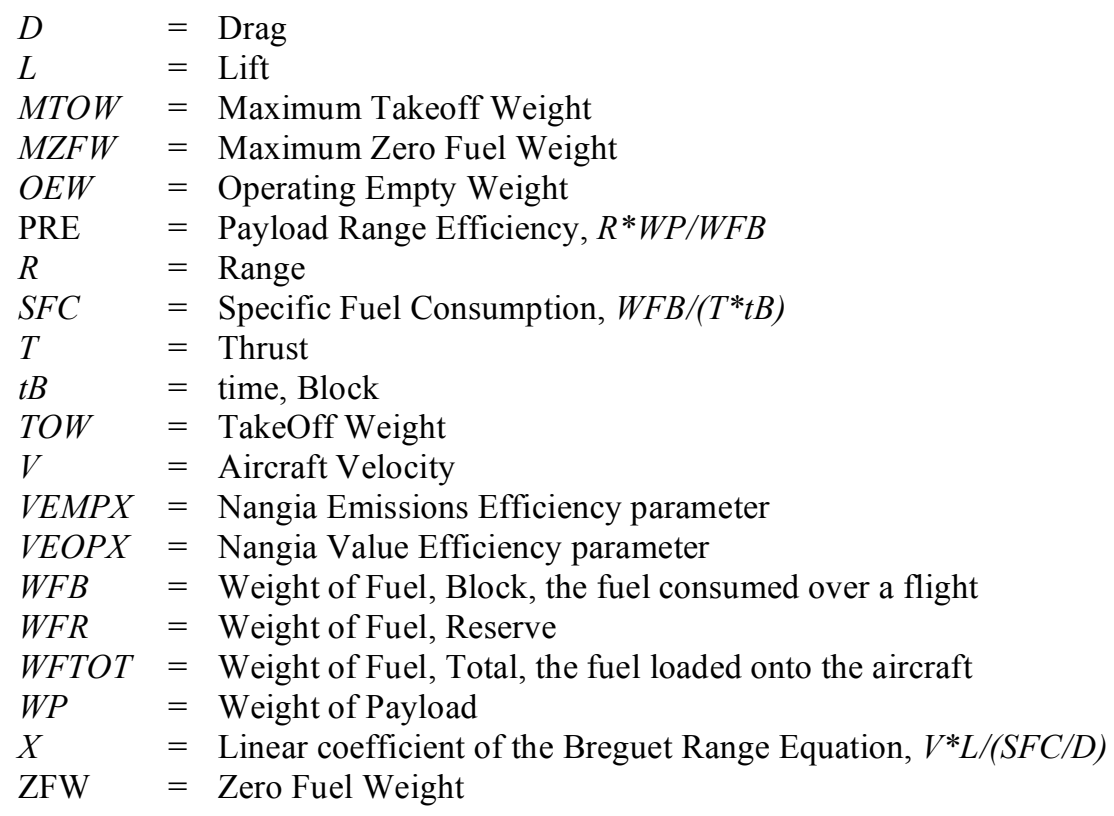

\section{Introduction}

CONSENSUS is building that both aircraft manufacturers and airlines are facing an ever more difficult future. A Dramatically rising fuel prices and the possibility of new regulations to limit Global Climate Change are altering the balance of priorities that have shaped the airliner's design and operation until now. These changes are driving a fresh reassessment of the characteristics desired when providing long-range air transportation. Recently, the possibility of altering both the design and operation of large airliners away from the historical trend of ever increasing stage lengths to that of a series of short stages has been proposed.

In his paper titled "Efficiency Parameters for Modern Commercial Aircraft,"1 R.K. Nangia builds upon the work of J.E. Green ${ }^{2-3}$ and proposes two main hypotheses. The first is that approximately half of the fuel used when flying long ranges in one stage can be saved if the trip is broken into three stages and the aircraft is redesigned for this

\footnotetext{
${ }^{*}$ Aerospace Engineer, Aeronautical Systems Analysis Branch, Mail Stop 442, AIAA Associate Member.
} 
operation. The second is that a new metric, or figure of merit, should be used to ensure that aircraft designs reflect the coming reprioritization of desired characteristics.

This paper will examine both hypotheses using significantly different methodologies and data sources and will present all of the results in context.

\section{Staging Long-range Airline Service}

There has always been a demand for very long-distance travel. For most people throughout history, the cost and inconvenience of the travel modes available at the time has meant that this demand has gone mostly unfulfilled. However, as the relative cost and average speed of the available travel modes have improved, more people have chosen to take these long trips. When the aircraft was first introduced, the technology was too immature to meet any significant demand. The aircraft were not capable of carrying passengers far enough, fast enough, or economically enough to entice many people to choose them over other available modes or even to make the trip at all. Aircraft technology improved rapidly, but still, there were short periods of time when the technology didn't allow for single stage service and airlines were willing to make significant operational concessions to enable very long-range travel.

While there are many historical examples of multiple stage service up and down the American continents, one could argue that each stop along the way was a destination in and of itself, and so doesn't technically count as staging.

However, a clear example case of staging, where a minority of stages could be justified as demanded travel, has occurred. In 1935, Pan American Airlines instituted their "China Clipper" passenger and airmail service from San Francisco, CA, to Hong Kong, China. ${ }^{4}$ The Martin M-130 flying boat was nowhere near capable of making the trip in one stage, and the obvious option of following the continental coastline added so much distance that both time and cost were unappealing. Fortunately, the Pacific Ocean does have a few small islands at regular intervals, more or less along a line from San Francisco to Hong Kong. Pan American absorbed the cost of building and operating service bases at these remote islands in order to capture the available demand until the start of WWII forced the cessation of operations. The route had six stages, namely San Francisco to Honolulu, to Midway, to Wake, to Guam, to Manila, and finally to Hong Kong. This operation shows that an airline is willing to adopt even extreme forms of staging if it makes economic sense. During the intervening war years, aircraft technology improved dramatically, making this kind of operation unnecessary to service the demand.

Another example of the extremes that airlines are willing to consider is the British Shorts company's attempt to build a staged airliner for Imperial Airways. ${ }^{4}$ The Atlantic Ocean does not have the evenly spaced islands that the Pacific Ocean does, making the option of landing to refuel much less attractive. Instead, Shorts designed and tested the Mercury-Maia composite aircraft, which was literally a small seaplane attached to the top of a much larger flying boat. The Maia would takeoff with the Mercury attached above and give the Mercury a good flying start so that it would be able to begin the trip with a useful load with which it was physically incapable of taking off. Unfortunately, the Mercury-Maia operation proved uneconomical and was abandoned. It wasn't until the Boeing 314 Clipper came into service that there was an aircraft capable of crossing the Atlantic Ocean with the right combination of speed, range, and economy, but it did so in a single stage.

Clearly, airlines are willing to consider unusual operations, including staging, if it captures travel demand at a profit. Currently, there are few staged airline operations, and these are relegated to only the longest of routes, such as London, UK to major cities in Australasia or New York, USA to cities in India.

Nangia correctly observed that the historical trend has been towards ever greater stage lengths. Jenkinson reports that the average international stage length had increased steadily by a total of $84 \%$ between 1960 and $1990 .^{5}$ This trend appears to be driven by fundamental market forces, and so will probably continue until the ultimate goal of single stage to any city pair in the world is a reality.

Both Green and Nangia have suggested that the new realities of high fuel prices and Global Climate Change are making this kind of operation unsustainable and that the strategy of staging long-range service can save a great deal of fuel as well as the emissions that come from burning it.

\section{A. Analytic Analysis and Results}

Both Green and Nangia have developed analysis methods that are based on the Breguet Range Equation. I won't go into detail about the analysis here, as the original papers do a much better job of outlining it than I would. ${ }^{1-3}$ What is important to understand about their methods is that they rely heavily on very coarsely correlated historical data, non-dimensionalization to reduce variation due to scale, and averaged values to reduce variation due to state changes over time. This is a classic analytic approach that seeks to make an analysis tractable by reducing the data and computational resources required. 
Both applied their analytic methods to the question of fuel efficiency as a function of range, on a hypothetical 479 passenger aircraft required to complete a total trip distance of $15,000 \mathrm{~km}$. The analysis was done for aircraft designs that performed both one stage and three stage operations. Each aircraft was specifically designed for the stage lengths required, specifically 15,000 and $5,000 \mathrm{~km}$ respectively.

Green's analytic method indicated that making the trip in one stage, instead of three, required Operating Empty Weight $(O E W)$ to increase by $41 \%$, TakeOff Weight (TOW) to increase by $76 \%$, and Weight of Fuel, Block (WFB) to increase by $40 \%$. These are relative measures, which illustrate important relationships, but it is also illustrative to have some absolute measures for further comparison as well. The metric used by Green and Nangia, Payload Range Efficiency ( $P R E$ ), is a good measure of fuel efficiency and, with a few unit conversion factors applied, is equivalent to the commonly used Seat-Nautical Miles per Gallon metric. It is important to realize that the PRE is an average value over the stage length, and so is only accurate for the stage length in which it was calculated. Green's results for $P R E$ are that the single stage aircraft attained $5,581 \mathrm{~km}$, while the three stage aircraft attained $7,832 \mathrm{~km}$.

Nangia reported that he found a few errors in Green's analysis and corrected them in his own analysis. Nangia's analytic method indicated that making the trip in one stage, instead of three, required $O E W$ to increase by $126 \%$, TOW to increase by $125 \%$, and $W F B$ to increase by $103 \%$. Nangia's results for $P R E$ are that the single stage aircraft attained 3,944 km, while the three stage aircraft attained 8,000 km. Figure 1 shows both Nangia's and Green's results for $P R E$ as a function of aircraft design range.

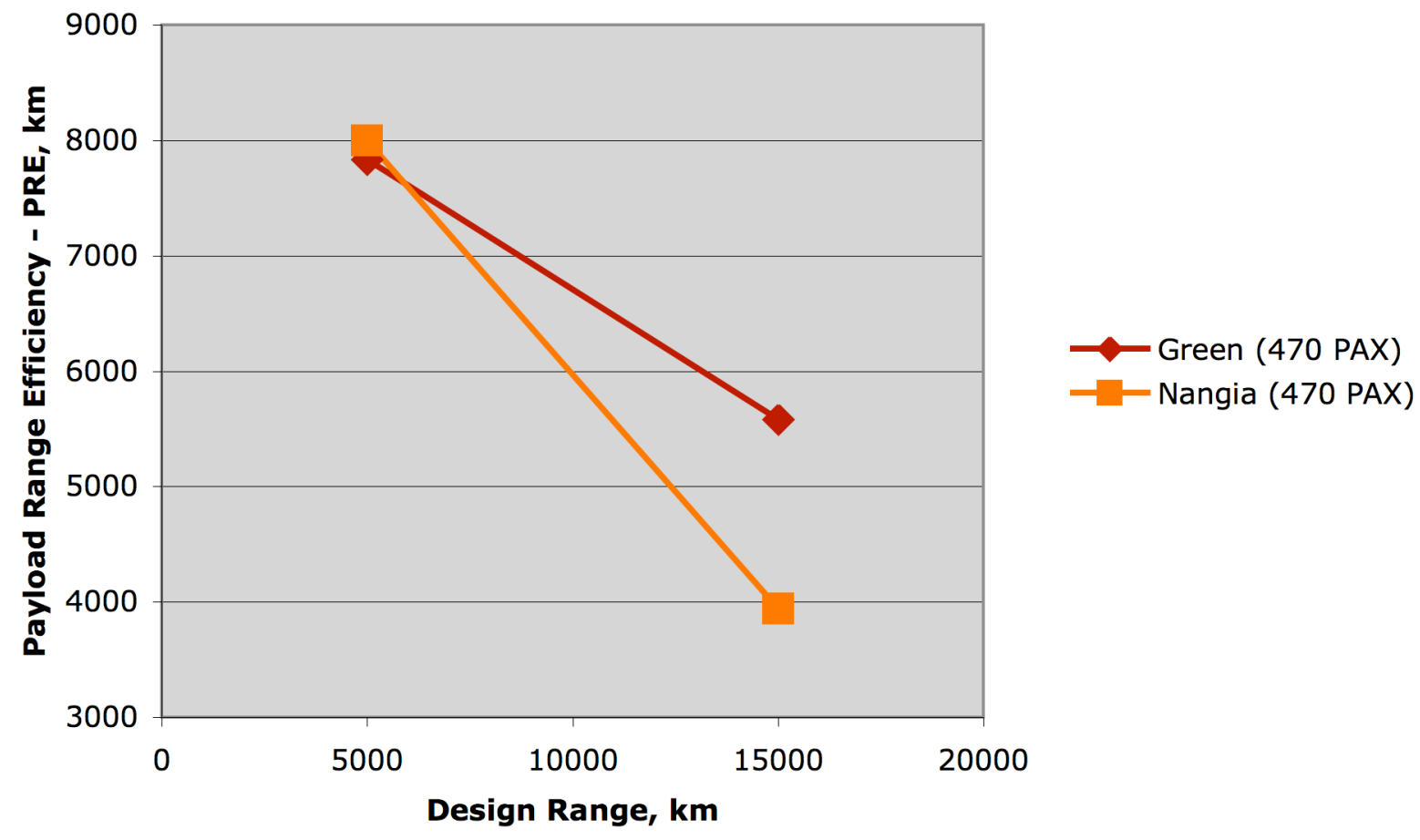

Figure 1. Payload Range Efficiency as a function of Design Range from Green and Nangia.

Despite some disagreement about the actual values of the single stage penalty, both analyses indicate that the penalty is substantial. If correct, these results indicate that changing the design and operations of large, long-range airliners to multiple stages would have a large positive impact on fuel consumption, operational cost, and emissions. The larger this impact is, the more likely that any negative aspects of staging would be more than outweighed; so accurate quantification is crucial.

\section{B. Numeric Analysis and Results}

I applied the standard conceptual design approach used at NASA Langley for the evaluation of new technologies, configurations, and operations of aircraft. This involved collecting publicly available reports and data, formulating the analysis plan, choosing a validation aircraft for tuning the analysis to an accepted standard, redesigning to a baseline aircraft from which to base relative comparisons, performing many redesigns to populate a 
database, understanding the results in the database, redesigning to correct mistakes, and finally reporting out the results with interpretations that are relevant for the intended audience.

The method used for redesign is a computer program named FLOPS (Flight Optimization System) ${ }^{6-8}$ It is an aircraft design synthesis code that breaks historical data and engineering methods down to fine detail and captures the multi-disciplinary interactions of these analyses through a time step integration over a mission. FLOPS has the ability to optimize an Objective function subject to equality and inequality constraints through a list of design variables under the designer's control. This is a capability far beyond simple resizing. Resizing is the act of converging to a new, self-consistent design. For any given set of requirements, there may be many such designs possible. Constrained optimization is a process by which the design space is explored and the best design, as defined by the Objective function, is reported out as the solution, while ensuring validity through the specified constraint set.

This numeric analysis approach has several advantages over the analytic approach besides the ability to optimize. The single biggest advantage is that the simplification through non-dimensionalization and averaging takes place at the lowest levels in the analysis, which allows a much more complex recombination of characteristics to be analyzed. With the analytic approach, the method tends towards overgeneralization because the details that determine the values are obscured. The values are only valid over the range of variation of the vehicles that were used to determine them and frequently the designer is unaware of the ranges of validity. In cases where new vehicles are being evaluated, if the characteristics, or any combination of characteristics, fall outside of the validity range of the database, then there is a real possibility that the analysis will yield incorrect results. The numeric approach, on the other hand, still suffers from some of the same database dependencies as the analytic methods, but by breaking the analysis down into much smaller and more detailed pieces, the numeric approach is able to assemble the results to cover combinations of parameters that do not exist in the original databases. Another advantage is that the detail available makes the result more transparent. Knowing why an answer is coming out the way that it is can be as important as knowing what the answer is. Having the finer detail available aids both in assessing the validity of the answer as well as understanding what may be driving the answer.

While the type of approach used will not guarantee that any given answer will be accurate, I believe that the numeric approach provides the best chance at correctly answering questions such as what are the potential benefits of staging airliner service?

\section{Research and Planning}

The hypothesis that there are large benefits from the staging of long-range airliner service has been most prominently espoused by Green and Nangia. Since all analysis methods and models are simplified representations of reality, they inevitably contain errors and comparing results from different analyses can be inconclusive. I did not want the veracity of this study to hinge on the reputations of the individuals or the heritage of the methods. Instead, I felt that the core of their hypothesis could be best approached by determining a minimum benefit using methods with no modeling error, then tuning an existing conceptual model to minimize modeling error, accepting the validation vehicle as the baseline vehicle, and finally performing a series of redesigns for populating the database. In this case, using published data for existing aircraft required no modeling, and so was free from modeling error. The computer model that I used for the redesign cases did have error, which I then minimized through calibration.

\section{Validation Vehicle}

After identifying that the characteristics desired for the study were the capability of very long-range operations and carrying a large payload; and deciding that a modern twin-engine configuration would be most relevant, I chose the Boeing 777-200 with the High Gross Weight Option model of airliner for validation. While smaller than the 470 passenger vehicles chosen by Green and Nangia, the B777-200HG is capable of carrying 305 passengers in a 3-class configuration the full $15,000 \mathrm{~km}$. Since it is at Maximum Takeoff Weight (MTOW) at this condition, the B777$200 \mathrm{HG}$ can be considered a near-optimal, modern, twin-engined validation aircraft for the analysis at this stage length. Unfortunately, the B777-200HG is substantially over designed for the 5,000 km range, making it a poor choice for validation at that stage length. There weren't any aircraft available at this short range that met all of the traits necessary for a validation aircraft. The closest was the Boeing 737-900. The B737-900 is a significantly different aircraft, being more cramped (2 class seating), smaller, slower, and having a much less advanced engine. Still, it is almost capable of making the $5,000 \mathrm{~km}$ range with 177 people on board and represents a real data point for an airliner designed to this short range. While it can't be considered a validation aircraft, it can be used as a less conclusive, but significant reference aircraft. Figure 2 shows the $P R E$ for of these existing aircraft over the ranges of interest. It is important to note that there is no possibility of modeling error in these values. It is also important to note that these results are conservative as there is no redesign from one range to the next.

Figure 3 shows the payload/range diagram of the B777-200HG, along with the combinations of payload and range used. ${ }^{9}$ Similar diagrams were used for all of the Boeing vehicles in the study. ${ }^{10,11}$ 


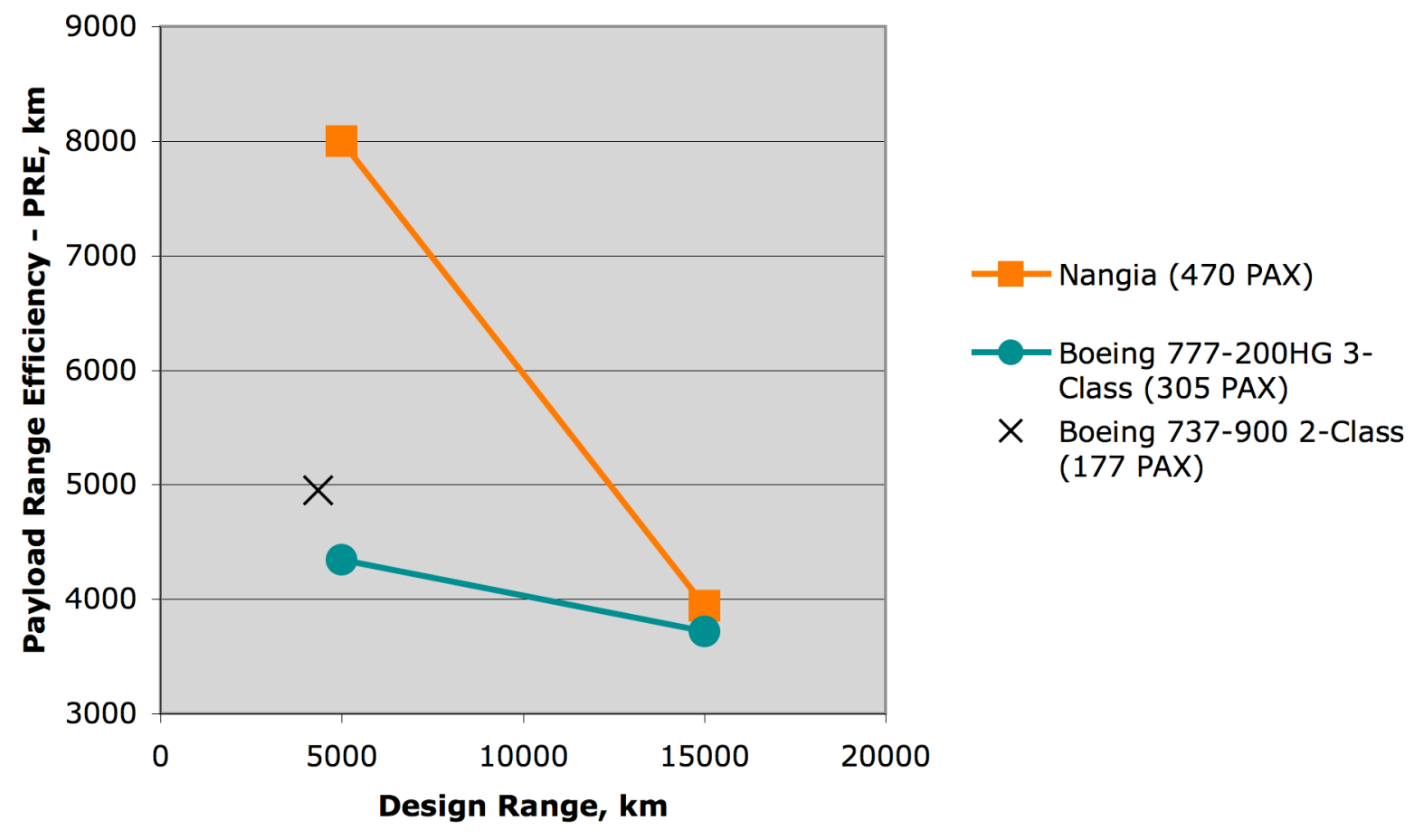

Figure 2. Payload Range Efficiency as a function of Design Range for Existing Boeing Aircraft.

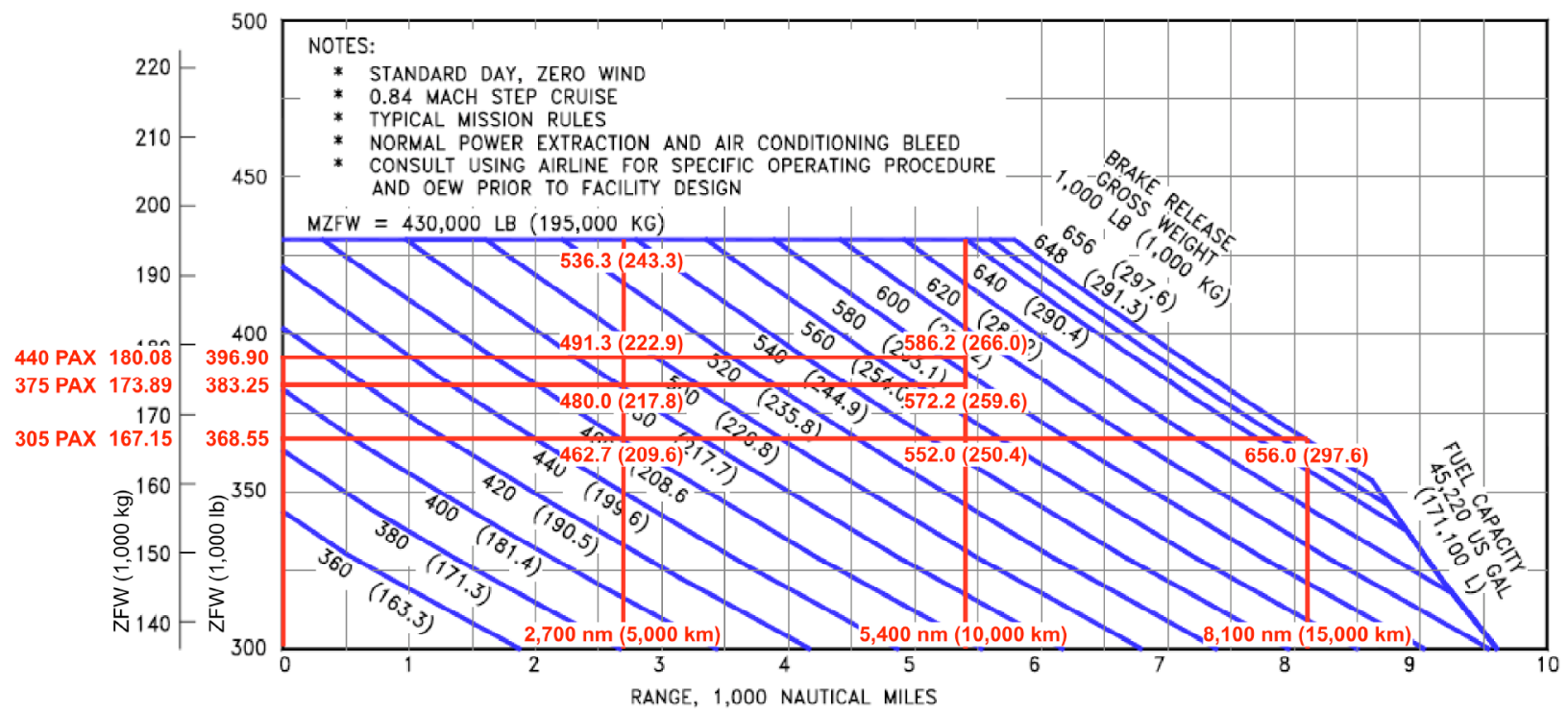

Figure 3. Payload as a function of Stage Length for the Boeing 777-200HG.

I utilized a FLOPS B777-200 conceptual design model that was created for a previous study. The model will execute an optimization where the Objective is to minimize TOW by modifying the design variables of wing area and engine thrust, while maintaining validity with many constraints. Examples of active equality constraints are constant payload weight and makeup, passenger comfort, stability and control, and technology level. There are many inactive inequality constraints, any of which may become active as the design space is explored, but the most important inequality constraint is the active second segment climb gradient that is required by Federal Air Regulations and is sizing the wing area and engine thrust. The model required minor tuning in order to accurately

American Institute of Aeronautics and Astronautics 
reflect the published Boeing values for TOW, OEW, and Weight of Fuel, TOTal (WFTOT). Given the lack of detailed weight, aerodynamic, and propulsion calibration data, it is not possible to accurately assign calibration factors to individual sources of error. This means that judgment needs to be applied to make the adjustments without significantly impacting the underlying trends during redesign. Two errors needed to be reduced during calibration.

The first error was that the model's OEW was approximately 1\% light, so I decided that distributing a 3\% increase across the wing, fuselage, tails, and landing gear weights, would be a neutral way of accounting for this error. This strategy was used because experience has shown that these items are more likely to be in error to this degree than the other items that make up the OEW and that distributing the error evenly would not unduly change the underlying weight trends.

The second error was that the model was consuming too much fuel. It is difficult to determine the source of the discrepancy, because there are several factors that can affect fuel consumption, and the lack of reliable detail data meant that the cause could not be accurately isolated. Rather than leaving the error in, I decided that reducing the overall fuel consumption estimate by $7.7 \%$ was the best strategy available. Since this correction represents a slope change, it does alter the underlying trend somewhat, but experience has shown that this is the best way to calibrate regression models.

With these two changes, the FLOPS model matched the validation vehicle very well.

3. Baseline Vehicle

In most studies, the validation vehicle can't be used as the baseline for comparison because it doesn't meet mission requirements and/or doesn't have the right level of technology. This usually means that the validation aircraft is redesigned in a series of steps to become an optimal and valid design for the mission requirements and technology timeframe specified. In this study, the validation vehicle is perfectly adequate to answer the question at hand and fits the range and technology level that both Green and Nangia used. There is a small issue in that my model only carries $65 \%$ of the passengers, which does introduce inconsistency due to scale effects.

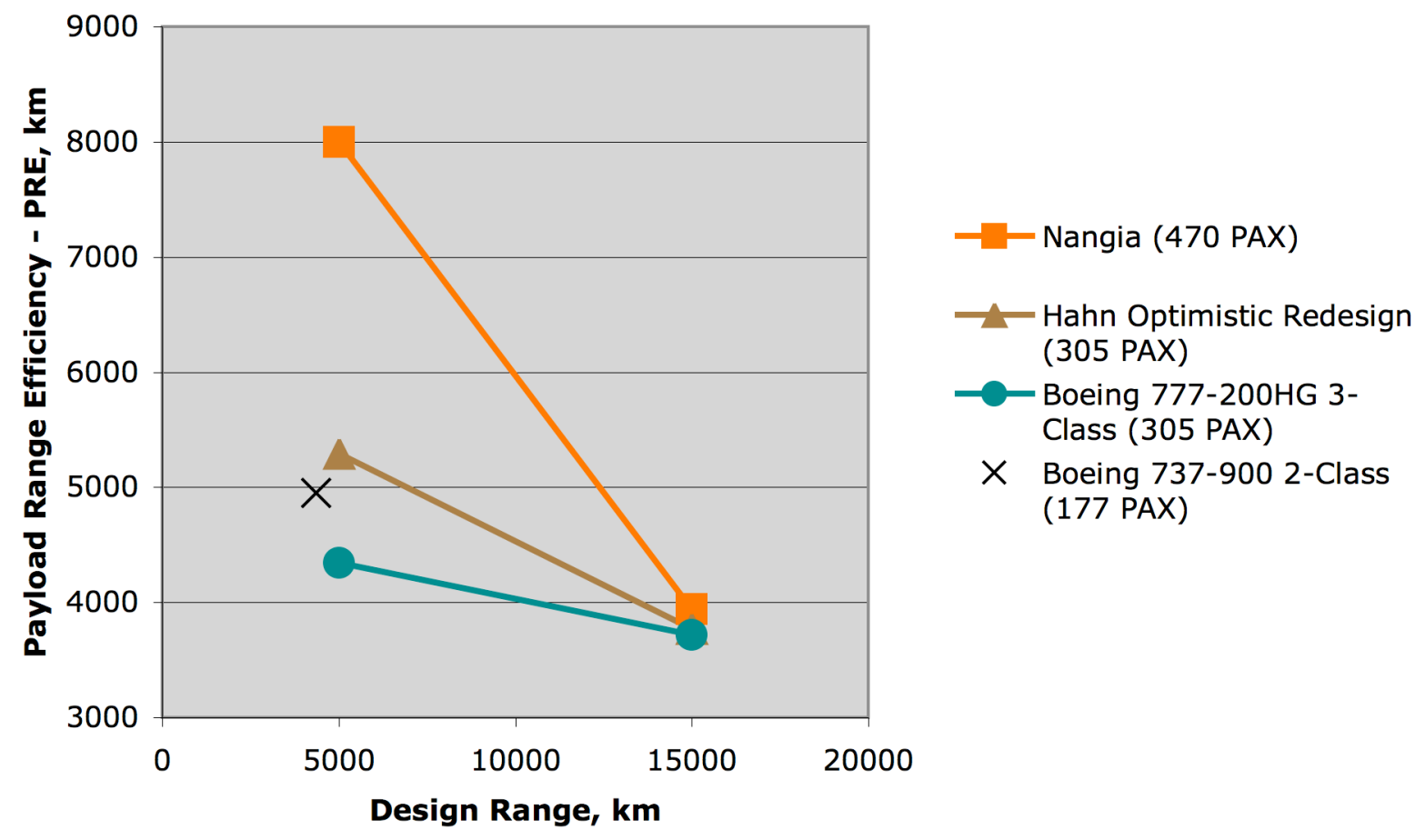

Figure 4. Payload Range Efficiency as a function of Design Range for Hahn Optimistic Redesigned aircraft.

\section{Populating the Database}

The first task was to evaluate the Boeing $777-200 \mathrm{HG}$ over the ranges desired to establish the minimal benefit available from staged operations using existing aircraft. Using the published $O E W$ and calculating the Weight of Payload $(W P)$ based on the number of passengers and $95.3 \mathrm{~kg}(210 \mathrm{lb})$ for each passenger with baggage, the Zero Fuel Weight $(Z F W)$ can be calculated. Where the $Z F W$ line crosses each range line, a $T O W$ can be found from the payload-range diagram (Fig. 3). The difference between $Z F W$ and TOW is the Weight of Fuel, Total (WFTOT), 
which includes the Weight of Fuel, Reserve $(W F R)$. Since PRE is calculated using the $W F B$, the $W F R$ must be subtracted from WFTOT. The payload-range diagram does include reserve fuel in the TOW, but doesn't state what the $W F R$ is. I chose to use the $W F R / M T O W$ regression from Nangia, which appears to be approximately constant at $4.4 \%$, to estimate $W F R$ for each stage length. The $P R E$ was then calculated at each stage length. (Fig. 2)

The second task was to establish any additional benefit available from a redesign from the high-confidence baseline. I performed an optimal sensitivity using FLOPS at design ranges of 5,000 and 15,000 km. An optimal sensitivity is when an individual constraint is changed and the vehicle is re-optimized. This analysis yields better results than the common sensitivity practice, because a new best design is found while maintaining validity through maintaining all constraints. The common sensitivity practice is merely a resizing of the design, which does not guarantee validity or optimality. Figure 4 shows that after calibration, the Hahn Optimistic Redesign 15,000 km aircraft has the same PRE as the validation aircraft, as intended.

Figure 4 also shows that the redesigned $5,000 \mathrm{~km}$ aircraft is an improvement over the clearly non-optimal Boeing 777-200HG, which is as expected. It is also somewhat of an improvement over the reference Boeing 737900. While a welcome outcome, there is no obvious relationship that should exist, so no definite conclusion can be made from this observation other than to say that it is in the reference's neighborhood.

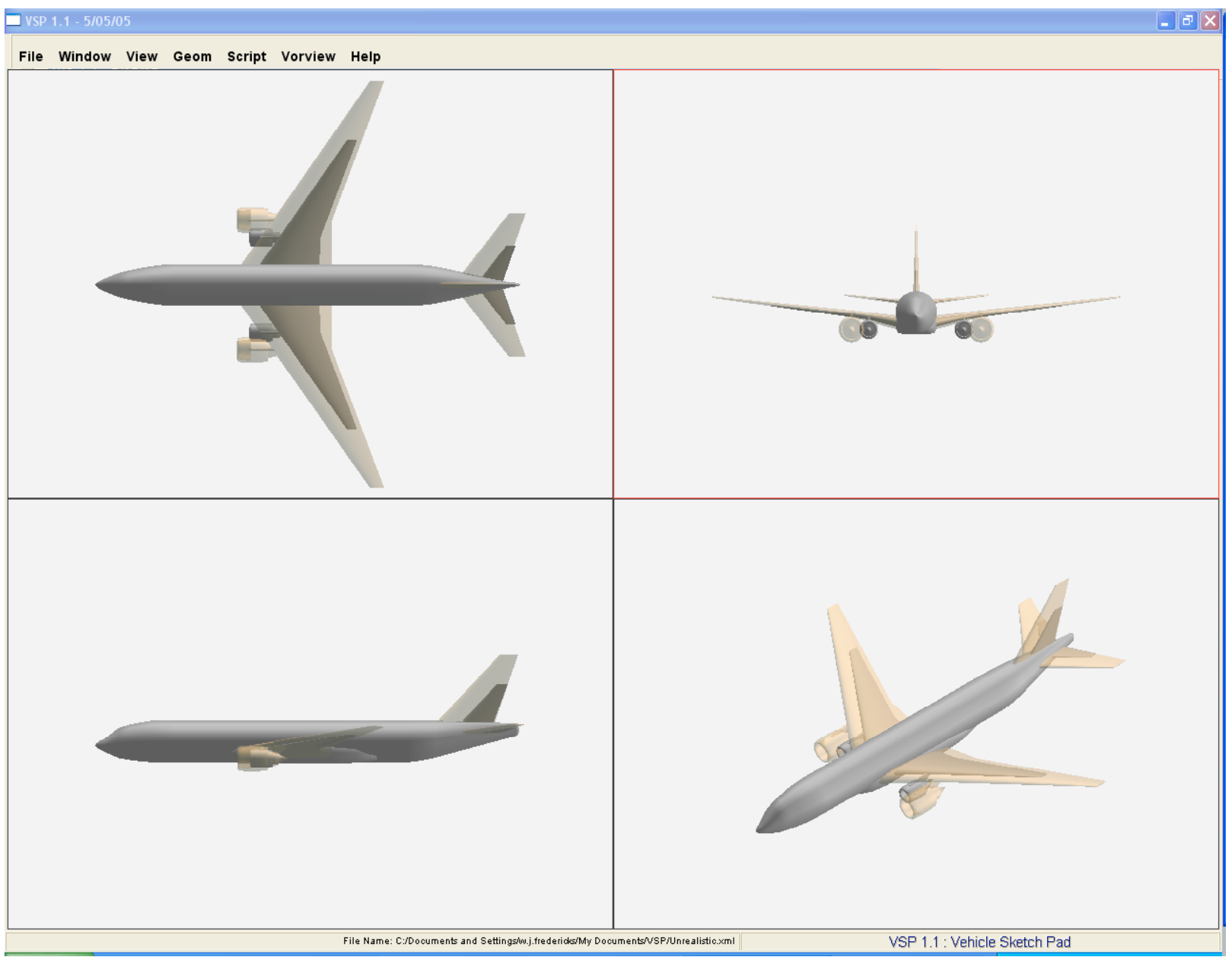

Figure 5. Four View Geometry Comparison between Boeing 777-200HG and Hahn Optimistic Redesign aircraft.

\section{Understanding Results}

After examination, I noticed that there was a problem with the model that became obvious only at the short stage length. The model uses a fixed set of Tail Volume Coefficients to size the vertical and horizontal tails. These coefficients are much more sensitive to changes in wing geometry than fuselage geometry, and since the fuselage geometry was fixed, the tails were tracking the wings as they shrank. Even a cursory examination reveals that this is an unrealistic behavior and that the optimization was being more strongly driven to small wings and tails than it 
should have been. (Fig. 5) This meant that the potential benefits through redesign were being significantly overstated. This design was overly optimistic, and so will be referred to from now on as the Hahn Optimistic redesign.
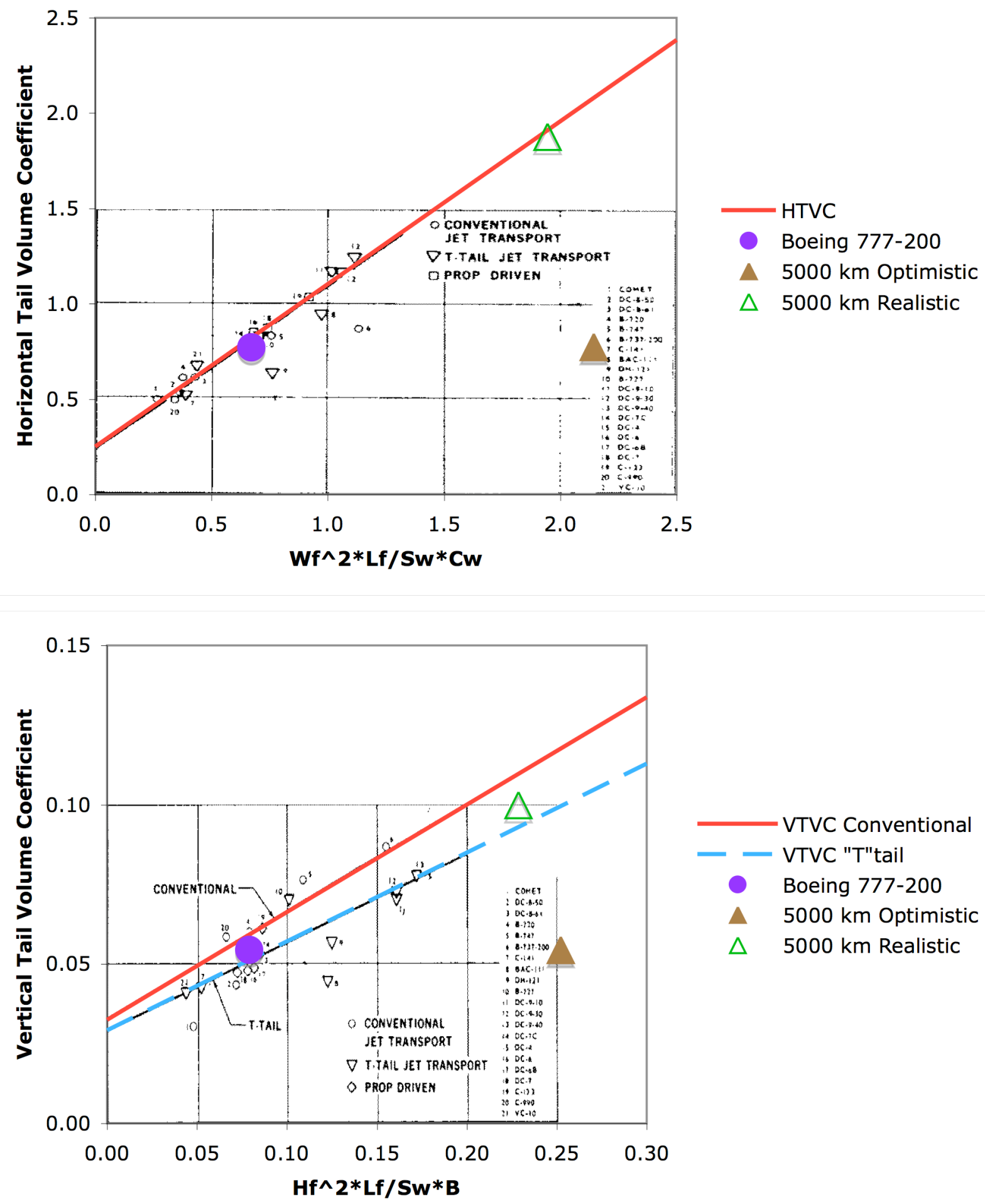

Figure 6a,b. Morris and Ashford Tail Volume Coefficient Correlation with Geometry Parameter. 


\section{Correcting Errors}

This unrealistic tail sizing is a common problem that can be addressed through a better method of conceptual tail sizing devised by Morris and Ashford of Douglas Aircraft. ${ }^{12}$ Figures $6 \mathrm{a}$ and $6 \mathrm{~b}$ show the Douglas method, which amounts to a correlation between Tail Volume Coefficient and a non-dimensional geometric parameter. As one can see, the validation aircraft, the B777-200HG, falls in line with the correlation, whereas the Hahn Optimistic redesign is far from acceptable. Through iterative manual sizing of the tails, I was able to bring the Tail Volume Coefficients into similar positions to the trend lines as the validation aircraft.

Figure 7 shows a top view of the Hahn Optimistic redesign on the left and the Hahn Realistic redesign on the right. The first item of note is that the tails on the Hahn Realistic redesign are indeed smaller than those on the validation aircraft, but not nearly as small as those on the Hahn Optimistic redesign. This is because, while the wing is substantially smaller, the fuselage has not changed at all and so it has the same polar moment of inertia and destabilizing influence that it had on the calibration aircraft, whose tails were sized by far more sophisticated methods and had passed certification trials. Tail Volume Coefficient is insensitive to these issues and so tends to undersize the tails. The second item of note is that the wing of the Hahn Realistic redesign is much smaller than that of the validation aircraft, but slightly larger than that on the Hahn Optimistic redesign. This is because the optimizer was able to reduce the Objective on the Hahn Optimistic redesign more by reducing the wing-tail combination, even if the wing was individually less efficient. Since this effect is greatly reduced on the Hahn Realistic redesign, the wing needed to be more efficient and so was made larger.

The scale of the impact on PRE from proper tail sizing was surprising. Using the B777-200HG at $5,000 \mathrm{~km}$ as the basis for $P R E$ without redesign, then the Hahn Optimistic redesign indicated that there was as much as a $26 \%$ improvement available for exploitation.

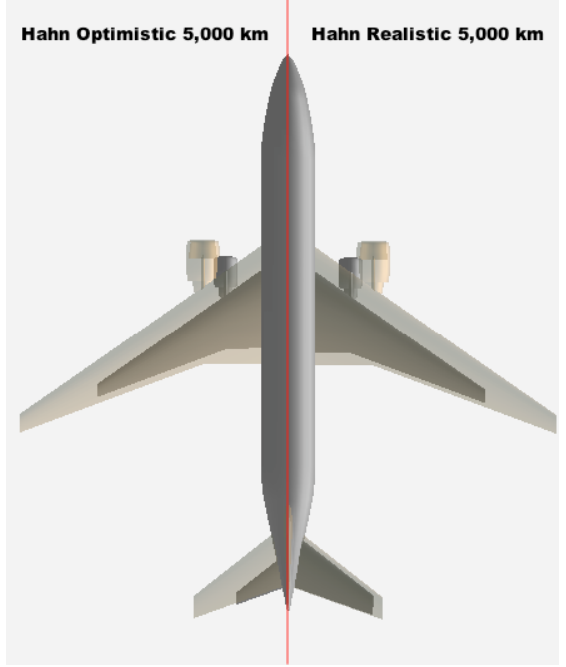

Figure 7. Top View Geometry Comparison between Boeing 777200HG, Hahn Optimistic Redesign, and Hahn Realistic Redesign aircraft. The Hahn Realistic redesign indicates that only $16 \%$ is actually available, which is a drop in potential of $38 \%$. (Fig. 8)

This example illustrates some of the advantages of the Numeric Analysis approach. The transparency promoted by the detailed buildup allowed a significant error to be caught, and the understanding provided gives confidence that the model is performing well. Also, the method is able to balance different characteristics that may not have existed in the original regression data in this proportion.

\section{Comparison Of Outputs, And Interpretation}

Using PRE as a metric for comparison across all three studies, it can be seen in Fig. 9 that there is a great deal of difference in opinion between Green, Nangia, and Hahn as to what the potential benefits are from staging.

The Boeing aircraft are the only aircraft considered in this paper whose predicted performance is free from modeling error. Since the B777-200HG can just meet the 15,000 km stage length at its $M T O W$, it can be considered to be nearly optimally designed for this combination of range and payload. At the $5,000 \mathrm{~km}$ range with the same payload, the aircraft is operating considerably below its $M T O W$ and so pays an $O E W$ and $W F$ penalty for having excess capacity that is not used. Even so, the benefit from staging is a significant $17 \%$ increase in $P R E$, due solely to the change in operation. 


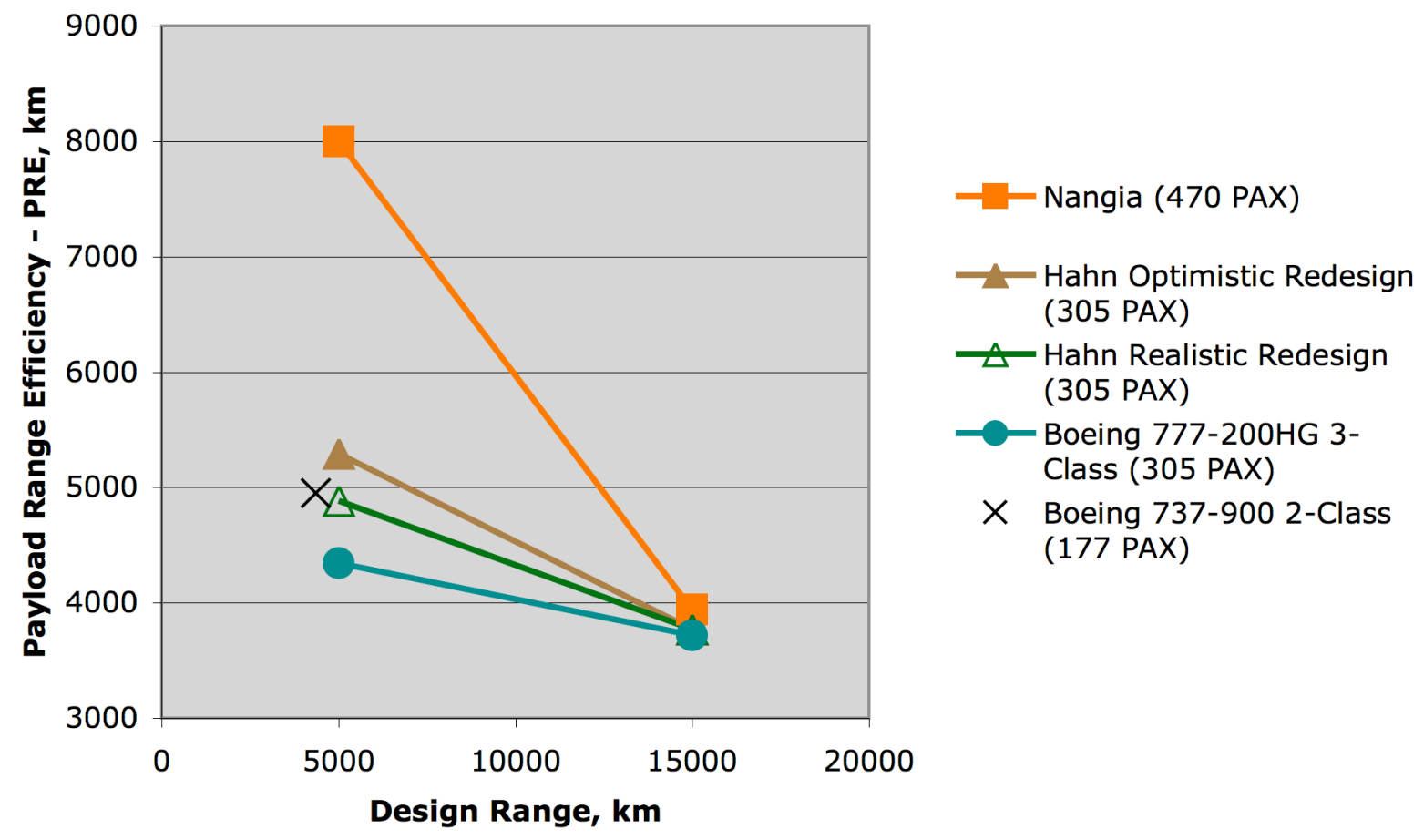

Figure 8. Payload Range Efficiency as a function of Design Range for Hahn Redesigned aircraft.

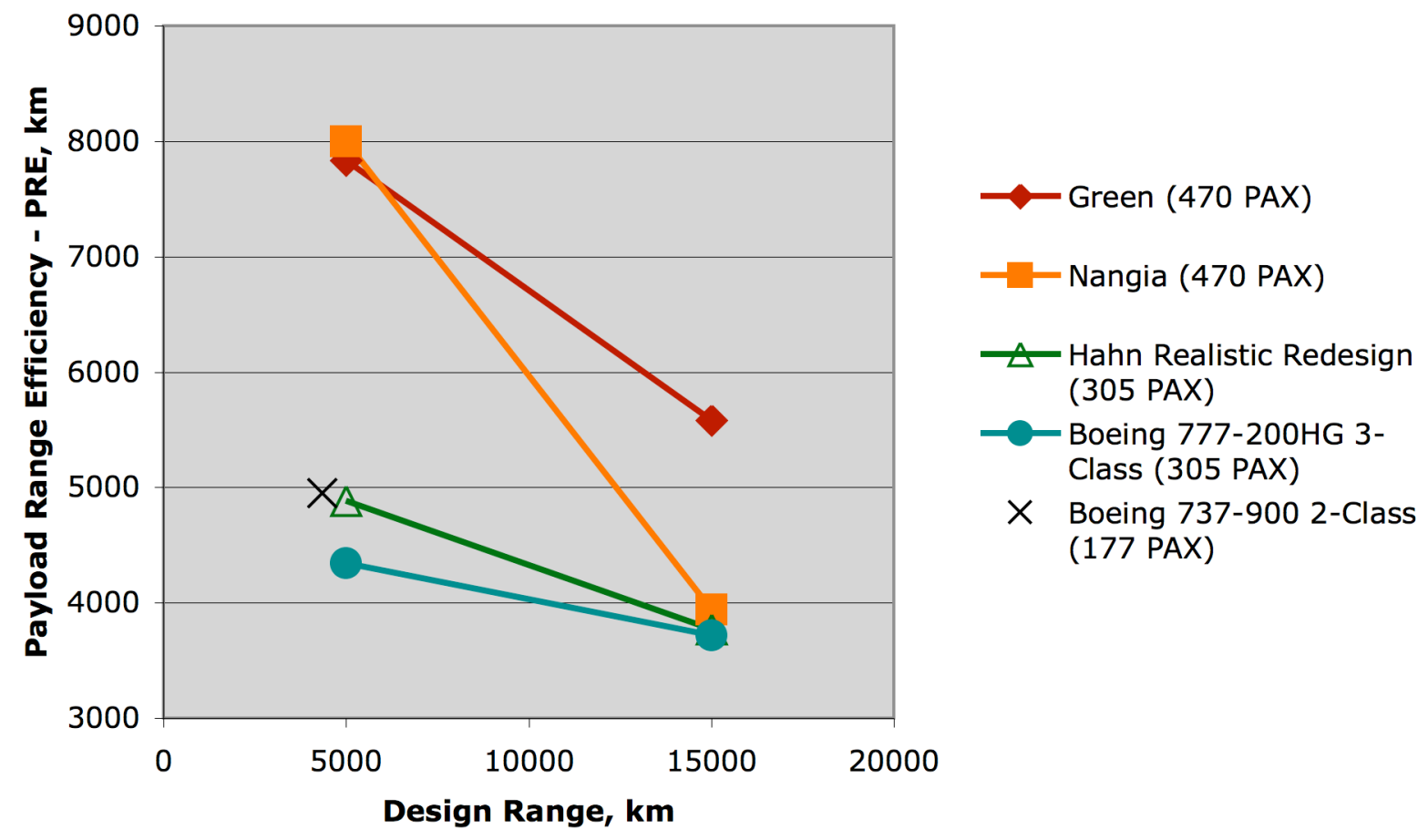

Figure 9. Payload Range Efficiency as a function of Design Range for All Three Studies. 
The Boeing 737-900 is included as another point of reference that represents a vehicle with no modeling error. It nearly meets the 5,000 km stage length at $M T O W$ and so can be considered to be nearly optimal for this stage length. There are several problems with making direct comparisons though. The Boeing 737-900 is a very different aircraft than the Boeing 777-200HG. It is much smaller and slower, has a less efficient engine, and is more cramped with 2 class accommodations. Still, one might assume that a Boeing 777-200HG class aircraft could do as well when redesigned optimally for the $5,000 \mathrm{~km}$ stage length.

The Hahn Realistic aircraft family uses a baseline that was tuned and optimally designed to be the same as the Boeing 777-200HG. By tuning the model, modeling error at this point is eliminated. While this tuning is no guarantee that there will not be significant modeling error introduced when redesigning to the shorter stage lengths, it does provide a solid anchor from which to proceed. Indeed, a significant modeling error was found in the Hahn Optimistic redesign and corrected on the Hahn Realistic redesign aircraft. While there is no conclusive proof that this design is accurate, it does have a PRE similar to the B737-900, which was as expected.

Nangia's $15,000 \mathrm{~km}$ stage length aircraft has a PRE that is quite similar to that of the Boeing 777-200HG validation aircraft, which is encouraging. However, Nangia's 5,000 km stage length aircraft has nearly double the PRE of the Boeing 737-900 reference aircraft.

Green and Nangia both agree on the maximum PRE attainable at 5,000 km, but disagree strongly at $15,000 \mathrm{~km}$. Given that Nangia has used a similar analysis to Green and has both identified and corrected errors in Green's analysis, I can only assume that these errors make Green's results unreliable.

This still leaves the large difference between Nangia's 5,000 km aircraft and the Hahn Realistic redesign unexplained. One potential source of error that may be inadvertently skewing the results may be an artifact of Nangia's historical regressions of weight fractions and technology constants. Given the extremely sparse data available for regressions, the tendency is to use it all even if it may constitute a wide range of technology levels or harbor hidden assumptions. As an example, short-range aircraft tend to be more densely loaded than long-range aircraft. This is because passengers are willing to endure lower comfort levels for short periods of time to get a lower ticket price, and the aircraft tend to carry cargo whenever conditions permit to maximize revenue. Figure 10 shows additional Boeing aircraft as well as loadings for the same aircraft to illustrate this point.

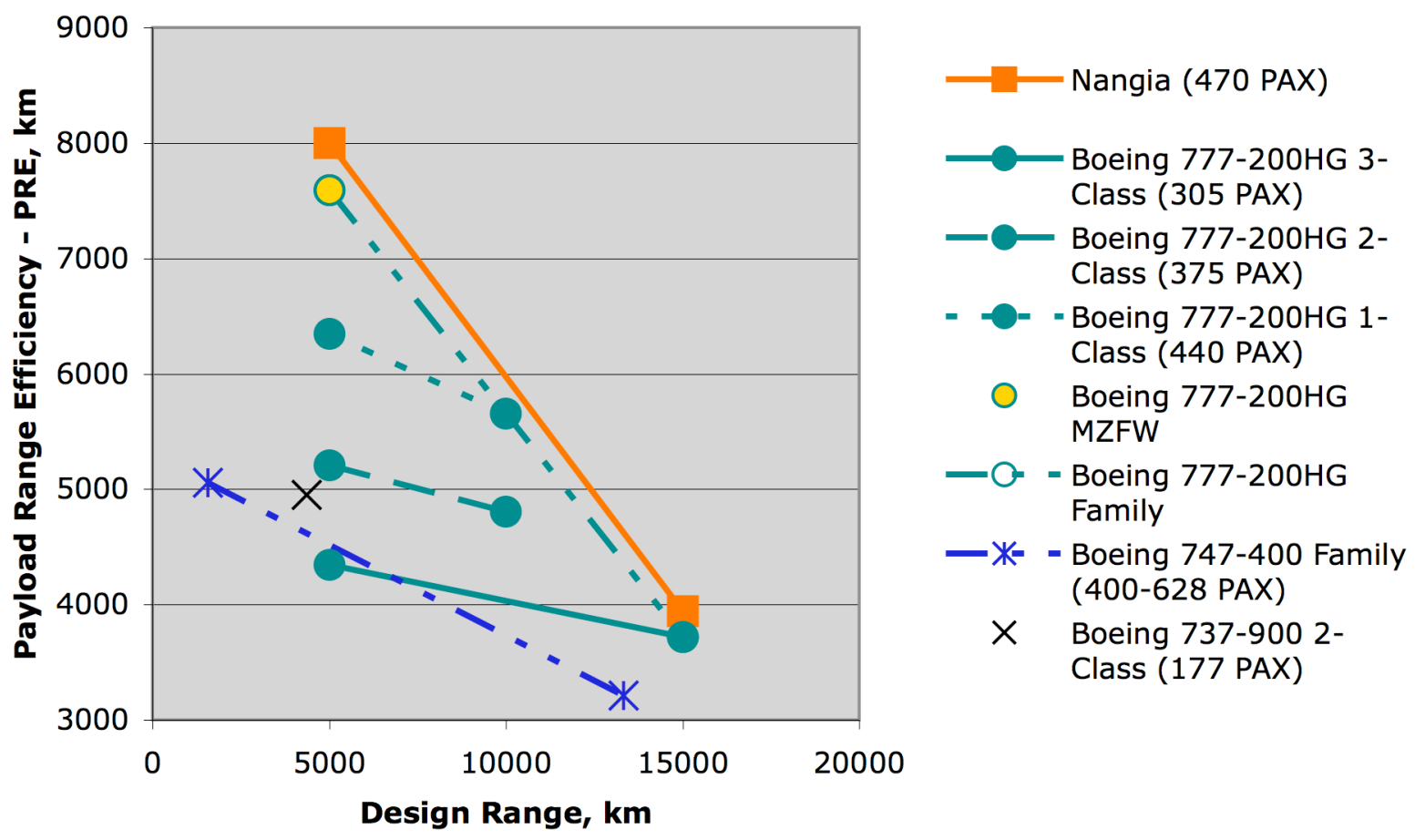

Figure 10. Payload Range Efficiency as a function of Design Range for Boeing Aircraft. 
The extreme example of varying loading to raise $P R E$ is the domestic variant of the Boeing 747-400, which can hold up to 628 passengers in 1-class accommodation. This strategy limits the maximum stage length to just 1,575 $\mathrm{km}$, but yields a good $P R E$ of $5,052 \mathrm{~km}$. The Boeing 747-400LR, on the other hand, holds just 400 passengers at a stage length of $13,334 \mathrm{~km}$, yielding a rather poor $P R E$ of $3,211 \mathrm{~km}$. It appears that the $\mathrm{B} 747-400$ has been pushed to the limits of its technology, which has exaggerated the PRE penalty to an exceptional $57 \%$ increase for single stage operation. (Fig. 10) Of course, the B747-400 was not chosen for the validation aircraft precisely because of these and other issues. For example, one wonders how the passengers would feel about being loaded onto that cramped aircraft for the ninth time to finish their $15,000 \mathrm{~km}$ trip.

The Boeing 777-200HG 2-class aircraft also illustrates that higher PRE is possible if the passengers are willing to sacrifice some comfort. While nowhere near as cramped as the B747-400 Domestic, this aircraft also shows a greater PRE than the Hahn Realistic optimally redesigned aircraft, which maintains the baseline's 3-class accommodation. This is an expected result. It can be shown that the denser an aircraft is, the more efficient it is. Passengers constitute a very low-density load, so any improvements here are likely to have a greater impact on PRE than anything else. Simply put, a kg of passenger has very different multi-disciplinary impacts on the aircraft than a $\mathrm{kg}$ of cargo or fuel. Since drag is proportional to wetted area, the denser cargo and fuel loads constitute less of a penalty to carry. Indeed, the wing usually has excess fuel volume available for all but the longest stage lengths, so parasite drag remains unchanged as fuel is added. Also, fuel is a particularly efficient mass to carry. Higher passenger and cargo weight is concentrated on the centerline, which raises wing bending moments. Fuel can be contained entirely in the wing, which reduces wing bending moments. While there is a tendency to trade these masses off one for one, this can add a high degree of error into the analysis that can lead to erroneous conclusions. Extending the trend to the 1-class loading, we see that the $P R E$ is improved still more, although significantly lower than Nangia's estimate. Taking this train of thought to its logical conclusion, I loaded the Boeing 777-200HG for a takeoff at a payload that yielded Maximum Zero Fuel Weight (MZFW). This loading is equivalent to that of 598 passengers and their baggage being somehow accommodated in the current fuselage. This unrealistic loading yielded a $P R E$ very similar to Nangia's estimate, which indicates that either his payload makeup (denser cargo being added) or his accommodation (passenger density) is changing as stage length changes. Because of the course nature of the high level regressions used, it is difficult to say what is driving the result.

While it is clear that this level of $P R E$ is attainable for short stage lengths, is this a valid result for this study? The only way to achieve the PRE predicted by Nangia at a fixed payload weight is to substitute denser cargo for passengers and/or reduce passenger comfort. It is undesirable for the airline to substitute low value cargo for high value passengers, as this would reduce revenue. It is undesirable for the passenger to endure more cramped accommodation because the total time spent on the aircraft is actually higher when staging than not. While the first stage may be bearable, each successive stage will become less so, unless the passengers are allowed to disembark at the end of each stage for a reasonable time period. Even so, the cumulative effect of spending more time on the trip and more time in the more cramped aircraft will probably more than offset any efficiency increase. Also, the greatest benefit only occurs at passenger densities that are illegal because egress requirements in the Federal Air Regulations could not be met.

Since this level of PRE is only possible if some of the original equality constraints of the study are violated, I feel that the results from the Hahn Realistic redesign are the most valid. The final results are: making the trip in one stage instead of three required $O E W$ to increase by $41 \%, T O W$ to increase by $82 \%$, and $W F B$ to increase by $29 \%$. The $P R E$ results are that the single stage aircraft attained $3,773 \mathrm{~km}$, while the three stage aircraft attained 4,883 km.

It is interesting to compare these results of redesign to those available through operation only. The Boeing 777$200 \mathrm{HG} 3$-class aircraft results are: making the trip in one stage instead of three required $O E W$ to increase by $0 \%$, TOW to increase by $42 \%$, and $W F B$ to increase by $17 \%$. The PRE results are that the single stage attained $3,715 \mathrm{~km}$, while the three stage aircraft attained $4,340 \mathrm{~km}$.

Therefore, approximately half of the total improvement to PRE from operating with three stages is due to the operational improvement and half is due to redesigning the aircraft to take advantage of this new operation.

It is also important to realize that this is an optimistic, academic study that assumes that usable airports are placed at even intervals along the flight path in still air. As any of these conditions varies from the ideal, the potential benefits from staging will erode.

\section{Negative Aspects of Staging}

These studies have focused on the benefits of staging airliner service with a very simple metric, $P R E$. There are also many reasons why staging is undesirable. While I have not quantified all of these negative aspects, they bear mentioning. 
First, there will be a negative impact on safety. While there are many hazards that the airline industry overcomes with stunning effectiveness, a great deal of their success can be traced to the fact that most airline flights fly above and avoid through route planning, most serious weather conditions. By requiring the airliner to descend through the lower atmosphere to a fixed location, the airlines lose this advantage. Also, the takeoff and landing phases are by far the riskiest phases of the flight, which are tripled by staging.

Second, total travel time is increased. Even if the system runs perfectly, this is a direct result of increasing the proportion of time spent in climb, descent, approach, taxi, and layover. The block time for the single stage aircraft is 17.18 hours, while the block time for the three stage aircraft is three times 6.00 hours, or 18 hours. Even considering unusual operations, the additional time to refuel would be at least 30 minutes per stage. For a three stage trip, this will add one hour to the block time, yielding a total of 19 hours. Experience with international flights has shown that layovers of three hours are common, while layovers of six hours are not uncommon. The potential for greatly increasing the trip time is clearly undesirable.

Third, terminal area noise and emissions pollution is increased. Since the staged aircraft is smaller, both its individual noise and emissions signatures are likely to be reduced, but not by a factor of three. Since the takeoffs and landings are tripled, terminal area pollution must go up significantly.

Fourth, schedules will be much more susceptible to unplanned delays. Airlines already have a great deal of trouble dealing with disruption due to weather, congestion, and mechanical trouble. All of these are more prevalent at the terminal areas than they are enroute. Tripling the takeoffs and landings will much more than triple the likelihood of delays from these sources.

Last, the staged aircraft will either be less durable or heavier and costlier than my estimate indicates. This is because one factor that I don't control for is the effect of fatigue cycles. Both the airframe and engine are susceptible to the number of load cycles that they experience. In the case of the airframe, the fuselage is pressurized and depressurized on each flight. For the staged aircraft, there are three times the cycles per trip and either the fuselage will have to be strengthened for this duty or its life will be only one third of the single stage aircraft. This is far from an academic theory. Island hopping airlines such as Aloha Airlines and JAL have experienced catastrophic fatigue failures in their fuselages. Also, the staged aircraft's wings will be exposed to the turbulence of the lower atmosphere more. This means that they will be put through more and greater magnitude load cycles, which will require structural reinforcement. Last, the engines of the staged aircraft will be subjected to more full throttle operation during takeoff, effectively tripling the number of cycles and length of time at full throttle per trip.

These serious issues all reduce the attractiveness of staging airliner service. The benefits of staging have to be substantially more important than these penalties for an airline and aircraft manufacturer to be willing to adopt staging as an operational model. It is unclear as to whether the idealistic $29 \%$ fuel saving of the Hahn Realistic redesign aircraft is enough to justify staging.

\section{New Design Metrics}

The other main hypothesis put forward by Nangia is that the new emphasis on cost and pollution requires new metrics to ensure balanced designs. Nangia correctly points out that $P R E$ is narrowly focused and proposed two new metrics that should be better. The first is the Nangia Value Efficiency parameter, VEOPX, which he claims is a measure where, "Higher value is better for lower structure weight, costs (acquisition and operating) and landing noise." The second is the Nangia Emissions Efficiency parameter, VEMPX, where, "Higher value is better for lower noise emissions, and operating costs." I will not comment on these claims, but a further investigation of VEMPX will highlight an important point.

A fundamental difference between the Analytic Analysis approach and the Numerical Analysis approach is not so much that one uses non-dimensionalization while the other doesn't, but rather the level at which the nondimensionalization takes place. The desire to non-dimensionalize is understandable; however, it should be understood that the very act of non-dimensionalization discards scale information in an attempt to find similarities to exploit. When done at a high level, the tendency is toward redundant information, rather than capturing new degrees of freedom.

When Nangia wanted to increase the degrees of freedom for his new design metric, he collected $P R E$, the linear coefficient of the Breguet Range Equation, and payload fraction to create the non-dimensional metric VEMPX,

$$
V E M P X=\left(\frac{P R E}{X}\right)\left(\frac{W P}{M T O W}\right)=\left(\frac{P R E}{X}\right)(\text { PayloadFraction })
$$


where PRE is defined in Eq. (2), $X$ is defined in Eq. (3), WP is the Weight of the Payload, and $M T O W$ is the Maximum TakeOff Weight.

$$
P R E=\frac{(W P * R)}{W F B}
$$

where PRE is the Payload Range Efficiency, $R$ is the aircraft Range, and WFB is the Weight of Fuel, Block.

$$
X=\frac{(V * L / D)}{S F C}=\frac{(V * L)}{S F C * D}
$$

where $X$ is the linear coefficient of the Breguet Range Equation, $V$ is the flight Velocity, $L$ is the aircraft Lift, $S F C$ is defined in Eq. (6), and $D$ is the aircraft Drag.

$$
\begin{gathered}
\mathrm{L}=M \mathrm{TOW} \\
\mathrm{D}=\mathrm{T}
\end{gathered}
$$

where $T$ is Thrust. Equations (4) and (5) are very common substitutions, which are valid under steady state, unaccelerated flight conditions such as cruise. Essentially, Eq. (4) sets lift equal to weight while Eq. (5) sets drag equal to thrust.

$$
S F C=\frac{W F B}{(T * t B)}
$$

where $S F C$ is the Specific Fuel Consumption, and $t B$ is time, Block.

$$
X=\frac{(V * M T O W)}{\left(\frac{W F B^{*} T}{T^{*} t B}\right)}=\frac{(V * M T O W * t B)}{W F B}
$$

Substituting Eqs. (4) - (6) into Eq. (3) and simplifying yields Eq. (7).

$$
\begin{gathered}
V=\frac{R}{t B} \\
\frac{P R E}{X}=\frac{(W P * R * W F B)}{(W F B * V * T O W * t B)}=\frac{W P}{M T O W}=(\text { PayloadFraction })
\end{gathered}
$$

Substituting Eq. (8) into Eq. (7) and simplifying yields Eq. (9). 


$$
\text { VEMPX }=(\text { PayloadFraction })^{2}
$$

Substituting Eq. (9) into Eq. (1) yields Eq. (10). VEMPX simplifies to the well-known non-dimensional metric of payload fraction, but in this case it is squared.

The preceding analysis illustrates how parameters that are non-dimensionalized at a high level may appear to have different degrees of freedom, but in reality often do not.

Instead of devising new non-dimensional metrics to capture the multi-disciplinary nature of balanced aircraft design, we at NASA have taken advantage of the optimization capability of our Numerical Analysis approach. The balance comes from the flexible interplay of a multitude of small disciplinary analyses, while subject to constraints, to minimize a specific Objective function. Historically, we have minimized $T O W$, as it had a strong correlation with Life Cycle Cost $(L C C)$, and this was the Objective used in this study. As the conditions that prompted Green and Nangia to pursue the possibility of staging, namely increasing fuel prices and concern about Global Climate Change, become a reality, the correlation between $T O W$ and $L C C$ is becoming less valid. I agree that a new Objective needs to be found that better represents the new reality. What we need is to find an objective with intrinsic value that takes into account the economic challenges that will drive airliner manufacturers' and operators' decisions to ensure that the technologies and operations proposed are attractive to them.

\section{Conclusion}

Two main Nangia hypotheses have been investigated. The first hypothesis, concerning the likely fuel consumption benefits from the staging of airliner service has been quantified, and my results indicate that using three stages for a total of $15,000 \mathrm{~km}$ would likely yield a $17 \%$ improvement from operation alone, a further $12 \%$ improvement from redesign for the 5,000 km stage length, resulting in a total possible improvement of $29 \%$. This result differs substantially from the previous work of Green and Nangia. While I can not be certain as to why this is, results using published Boeing payload-range diagrams indicate that the previous work may not have controlled for payload density, skewing their results.

While it is likely that staging airliner service can save a substantial amount of fuel, there are serious negative impacts that have not been quantified. The greater the fuel savings, the greater the likelihood that staging will overcome these negative impacts. Given that my best estimate is less than a third of the earlier estimates, the likelihood that staging will still be attractive is significantly diminished.

The second hypothesis, concerning the development of new, high level, non-dimensional metrics to promote more balanced aircraft design has been examined, and it is shown that there are inherent pitfalls to this approach. While non-dimensionalization is a useful technique when applied to low level analyses, there is a tendency towards seemingly unrelated metrics having redundant information at high levels. This tendency toward high level redundancy is shown through the specific example of the simplification of the proposed Nangia Emissions Efficiency parameter, VEMPX, to the well-known non-dimensional parameter of payload fraction, squared.

The basic approaches of Analytic Analysis and Numerical Analysis are compared on this specific study. Both approaches have strengths and weaknesses, but the transparency and flexibility of the Numerical Analysis approach promotes the discovery of errors and understanding of how parameters are driving the result as well as allowing for tighter control over the study's execution.

\section{Acknowledgments}

I would like to thank Fayette Collier for allowing me to spend the time and effort needed to produce this paper, Jeffrey Berton whose initial efforts gave me the kind of courage to commit that can only come from knowing the answer beforehand, and Philip Arcara for providing the original FLOPS model in my hour of need.

\section{References}

\footnotetext{
${ }^{1}$ Nangia, R. K., "Efficiency Parameters for Modern Commercial Aircraft,” The Aeronautical Journal, Vol. 110, No. 1110, 01 August 2006.

${ }^{2}$ Green, J. E., "Air Travel - Greener by Design: Mitigating the Environmental Impact of Aviation: Opportunities and Priorities," Report of the Greener by Design Science and Technology Sub-Group, URL: http://www.greenerbydesign.org.uk/ FILES/publications/GbD - 2005 Science and Technology Report.pdf [cited 04 September 2007].
} 
${ }^{3}$ Green, J. E., "Air Travel - Greener by Design: The Technology Challenge," Report of the Greener by Design Technology Sub-Group, URL: http://www.greenerbydesign.org.uk/ FILES/publications/GbD - 2003 The Tech Challenge.pdf [cited 04 September 2007].

${ }^{4}$ Gandt, Robert L., China Clipper, The Age of the Great Flying Boats, Naval Institute Press, Annapolis, Maryland, 1991, Chaps. 16, 19.

${ }^{5}$ Jenkinson, Lloyd R., Civil Jet Aircraft Design, American Institute of Aeronautics and Astronautics, Reston, Virginia, 1999 , pp. 5.

${ }^{6}$ McCullers, L. A., "Aircraft Configuration Optimization Including Optimized Flight Profiles, Multidisciplinary Analysis and Optimization - Part 1," NASA CP-2327, 1984.

${ }^{7}$ McCullers, L. A., and Lynch, R. W., "Dynamic Characteristics of Advanced Filamentary Composite Structures, Volume II, Aeroelastic Synthesis Procedure Development," AFFDL-TR-73-111, 1974.

${ }^{8}$ Arcara, P. C. Jr., Bartlett, D. W., McGraw, M. E., and Geiselhart, K. A., "Technology Benefits for Very Large Subsonic Transports," AIAA-93-1178, 1993.

9 Boeing, “777-200/300 Document D6-58329 (Revision C, July 2002)," 777 Airplane Characteristics for Airport Planning, URL: http://www.boeing.com/commercial/airports/777.htm [cited 04 September 2007].

${ }^{10}$ Boeing, "747-400/-400ER Document D6-58326-1 (Revision D, December 2002))," 747 Airplane Characteristics for Airport Planning, URL: http://www.boeing.com/commercial/airports/747.htm [cited 04 September 2007].

${ }^{11}$ Boeing, “737/BBJ Document D6-58325-6 (NEW, Oct 2005)," 737 Airplane Characteristics for Airport Planning, URL: http://www.boeing.com/commercial/airports/737.htm [cited 04 September 2007].

${ }^{12}$ Morris, J., and Ashford, D. M., "Aircraft Fuselage Configuration Studies Point to Use of Multideck Fuselages," Society of Automotive Engineers, SAE 670370, Warrendale, PA. 